\title{
Identification of New Molecules in the Trunk Bark of Sarcocephalus Pobeguinii
}

\author{
Ousmane Keita $^{1 *}$, Huguette Agnaniet ${ }^{2}$, Rokia Sanogo $^{3}$ \\ ${ }^{1}$ Department of Biological engineering of Institute of Applied Sciences (ISA) -University of Sciences, Technics \\ and Technologies of Bamako - Mali \\ ${ }^{2}$ Laboratory of Natural Products and Organometallic Synthesis (LASNSOM) - Faculty of Sciences of University \\ of Sciences and Technics of Masuku. Franceville, Gabon. \\ ${ }^{3}$ Laboratory of Pharmacognosy of Faculty of Pharmacy - University of Sciences, Technics and Technologies of \\ Bamako (USTTB) - Mali
}

*Corresponding Author: Ousmane Kéita, Department of Biological engineering of Institute of Applied Sciences (ISA) -University of Sciences, Technics and Technologies of Bamako - Mali

\begin{abstract}
:
\section{Background}

Medicinal plants are commonly used in both traditional and modern medicine. Sarcocepahalus pobeguinii is a plant used in the treatment of several diseases in Africa. Because of their uses, these plants contain therapeutic virtues often due to the different molecules present.
\end{abstract}

\section{Objective}

The purpose of this study is to identify new molecules of trunk bark of Sarcocephalus pobeguinii.

\section{Method}

$100 \mathrm{~g}$ of crushed bark from the trunk of Sarcocephalus pobeguinii were introduced into $1000 \mathrm{ml}$ of distilled water and then kept for 30 minutes at boiling point. The extract is injected into the Ultra Performance Liquid Chromatography coupled with a mass spectrometer. Detection of the extract results in the appearance of a peak whose retention time and surface area are then recorded. The molecules to be analyzed are bombarded by a laser beam and the positive, charged $(+z e)$ molecular fragments are then accelerated in an electric field. Measuring the mass/charge ratios $(\mathrm{m} / \mathrm{z})$ of the atoms or molecules present in a given sample has made it possible to obtain their molecular mass, identify them and determine their raw formula by comparison with a database.

\section{Results}

The analysis by UPLC made it possible to detect 50 peaks of which 4 were majority peaks with retention times of 4.27; 4.37; 4.52 and 5.78 respectively. The percentage of peaks 20, 21, 22 and 40 are 15\%, 9\%, 7\% and $12 \%$ respectively. On the other hand, the molecular fragmentation of the mass spectra of peaks 35, 37 and 40 of the detected compounds made it possible to identify tetrahydrodeoxycordifoline, quercetin and magniflorine with the respective raw formulae $\mathrm{C}_{28} \mathrm{H}_{36} \mathrm{O}_{11} \mathrm{~N}_{2}, \mathrm{C}_{15} \mathrm{H}_{10} \mathrm{O}_{7}$ and $\mathrm{C}_{20} \mathrm{H}_{20} \mathrm{O}_{3} \mathrm{~N}_{2}$

\section{Conclusion}

This study identified quercetin, magniflorine and tetrahydrodeoxycordifoline in the bark of the trunk of Sarcocephalus pobeguinii allowing the identification of other molecules.

Keywords : Sarcocepahalus pobeguinii, molecules, HPLC/MS

\section{INTRODUCTION}

Sarcocephalus pobeguinii (syn. Nauclea pobeguinii) is a tree $4-25 \mathrm{~m}$ high and $8-60 \mathrm{~cm}$ in diameter. It is common in backwater and riverbanks. Sarcocephalus pobeguinii, because of these virtues, is used both in food and in therapy [1].

The work noted in the literature concerns both total extracts or individual organs and isolated compounds demonstrating antimalarial $[2,3,4]$, antibacterial, antibiotic and antioxidant properties $[5,6]$. 
Numerous scientific works on the genus Nauclea highlight monoterpenes, saponins, flavonoids, alkaloids [7,8,9,10,10,11,12], steroids and glycosides [13,14]. The structures of the characteristic compounds isolated from the various organs belong mostly to the alkaloid family. For example, (5S)5-carboxystrictosidine, 19-O-methylangustoline, 3-O- $\beta$-fucosylquinovic acid, 3-ketoquinovic acid and strictosamide have been detected in the trunk bark [20]. While other alkaloids such as angustine, naufoline, angustoline, nauclefine, O-acetyl-angustoline, 3,14-dihydroangustine as well as two quinovic acid glycosides have been identified in the roots [15,16,17,18]. Naucleamides, nauclefolinin, angustin, angustifolin, nauclefin and naucletin, cadambin and naufolin, naucleidinal and epinauclédinal, strictosamide, 10-hydroxystrictosamide, nauclefolin and nauclechin were also isolated from stem bark and various other organs $[7,12,19,20]$.

\section{Material AND MethodS}

\subsection{Plant Material and Preparation}

The trunk bark of Sarcocephalus pobeguinii Hua ex Pellegr. harvested in Lambaréné (Gabon) in January 2010 and dried at room temperature for one week in the shade and protected from light and humidity. $100 \mathrm{~g}$ of crushed trunk bark of Sarcocephalus pobeguinii were introduced into $1000 \mathrm{~mL}$ of distilled water and kept boiling for 30 minutes.

\subsection{Analysis of the Extract by UPLC/SM/TUV}

UPLC (Ultra Performance Liquid Chromatography) is performed in RP (reverse phase) mode, and the chromophore detection mode is followed by the TUV detector for UPLC, and a time-of-flight (TOF) mass spectrometer is coupled to a tandem quadrupole (TQ) detector. Thus, the molecules to be analyzed are bombarded by a laser beam. The positive, (+ze) charged molecular fragments are then accelerated in an electric field. The ions formed will reflect the most stable ions that the molecule can form. The highest molecular weight peak in the spectrum will represent the parent molecule with one electron less which is called the molecular ion $(\mathrm{M}+)$.

Mass spectrometry is a chemical characterization technique that consists of measuring the mass/charge ratios $(\mathrm{m} / \mathrm{z})$ of atoms or molecules present in a given sample in order to obtain their molecular weight, identify them and determine their raw formula by comparing it with an online database.

The identification and quantification of the compounds in the extract and sub-fractions were performed using a UPLC-TUV-SM system.

\section{RESULTS AND DISCUSSION}

\subsection{High Performance Liquid Chromatography of the Extract}

Figure 1 shows the chromatogram of the extract.

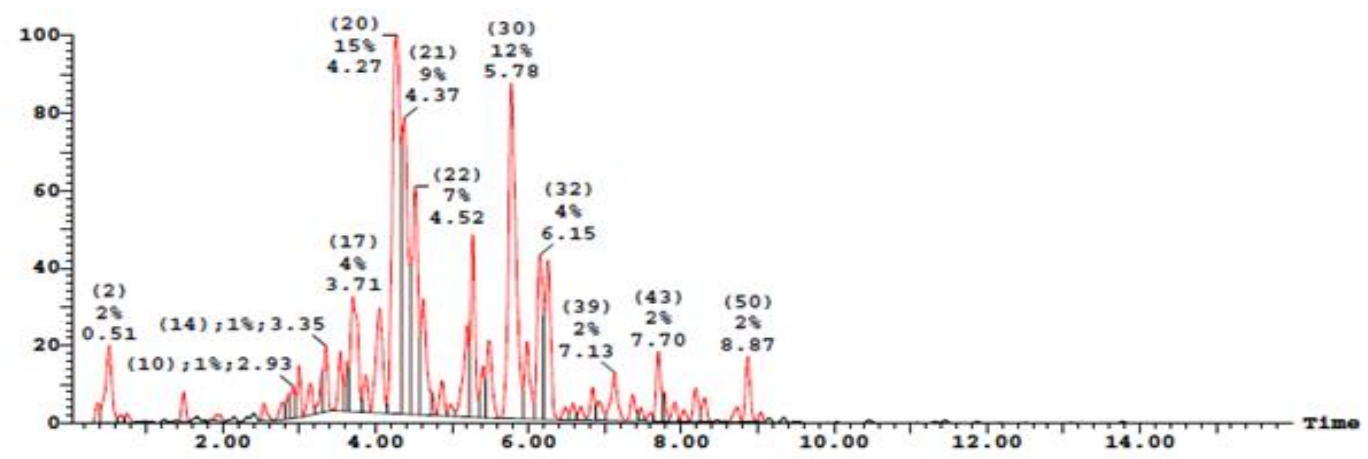

Figure1. Chromatogram of crude extract by UPLC

A total of about 50 peaks were detected, of which four were majority peaks above $7 \%$. These are peaks $20(15 \%), 21(9 \%), 22(7 \%)$ and $30(12 \%)$ which elute at retention times 4.27; 4.37; 4.52 and 5.78 respectively as shown in the chromatographic profile in Figure 2. 


\subsection{High Performance Liquid Chromatography Coupled with Mass Spectroscopy}

Molecular fragmentations of the peak mass spectra obtained by high-performance liquid chromatography have been listed in Table I.

Table1. Molecular fragmentations of the mass spectra of the different peaks of the detected compounds

\begin{tabular}{|c|c|c|c|c|}
\hline $\begin{array}{l}\text { Peak } \\
\text { no }\end{array}$ & $\begin{array}{l}\text { Retention } \\
\text { time }\end{array}$ & Percentage & {$[\mathbf{M}]^{+} \quad \mathbf{m} / \mathbf{z}$} & Molecular ion \\
\hline 1 & 0,36 & 0,37 & 214.9 & $\begin{array}{l}214.9(100), \quad 198.9(64), 336.8 \quad(45), \quad 122.9(42), \\
290.9(29), 458.8(25), 344.9(15),(542.7(13), 672.7(7), \\
862.6(3), 908.6(3), 1046.5(2)\end{array}$ \\
\hline 2 & 0.51 & 2 & 381.1 & $\begin{array}{l}\text { 381.1(100), 362.1(35), 351.1(21.5), 543.1, 258.1(13.5), } \\
\text { 443.1(11), 144.1(5), 191.0(5), 614.2(5), 857.3(1.5), } \\
704.2(3), 739.2(3)\end{array}$ \\
\hline 3 & 0.66 & 0,16 & 543.1 & 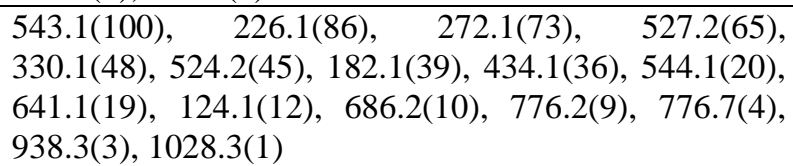 \\
\hline 4 & 0.74 & 0,1 & $\begin{array}{l}182.1 \\
\mathrm{M}-\mathrm{CH}_{3}\end{array}$ & $\begin{array}{l}\text { 182.1(100), 136.1(24), 272.1(20), 515.1(16), } \\
\text { 365.1(12.5), 543.1(9), 735.1(4), 641.1(3) } \\
\text { m/z 197 [M] }]^{+}=\square \text {-carbolinium } \\
\text { Specter UV = melinonine F }\end{array}$ \\
\hline 5 & 1.49 & 0,58 & 224.1 & $\begin{array}{l}224.1(100), \quad 224.1(12), 226.1(2), \quad 138.1(2), 358.1(4), \\
386.2(1), 920.3(1), 1001.3(1), 1163.4(1), 1244.4(1)\end{array}$ \\
\hline 6 & 1.92 & -- & 407.1 & 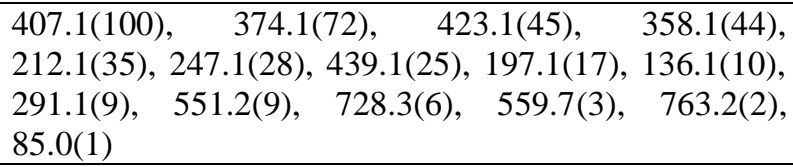 \\
\hline 7 & 2.54 & 0,21 & 521.2 & 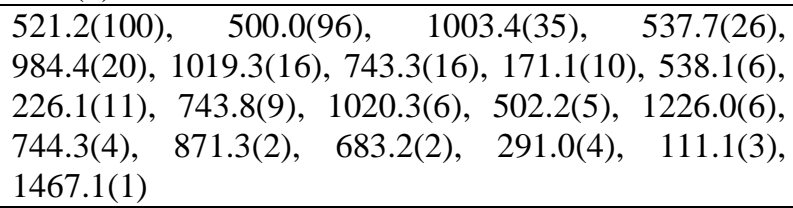 \\
\hline 9 & 2.87 & 0,73 & 513.2 & $\begin{array}{llll}513.2(100), & 593.2(68), \quad 500.0(36), & 692.2(41), \\
471.1(35), 468.1(30), 244.1(25), 692.7(20), & 594.2(19), \\
233.1(16), 251.1(15), 700.2(12), & 204.1(10), & 287.1(5), \\
951.3(5), 916.3(5), 154.1(3) & \end{array}$ \\
\hline 10 & 2.93 & 1 & 529.2 & $\begin{array}{llll}529.2(100), \quad 530.2(21), & 530.2(21), & 513.2(17), \\
692.2(12), 471.1(11), & 179.1(6), & 672.7(6), & 701.2(1), \\
916.3(1), 950.2(1.5) & & & \end{array}$ \\
\hline 11 & 2.99 & 0,93 & 575.2 & $\begin{array}{l}\text { 575.2(100), 576.2(28), 529.2(15), 609.2(2), 355.1(5), } \\
\text { 151.0(4), 208.1(4), 609.2(3), 402.2(2) } \\
\text { Pic 11 = 5-carboxystrictosidine, } \mathrm{C}_{28} \mathrm{H}_{35} \mathrm{O}_{11} \quad \mathrm{~N}_{2} \text {; } \\
{[\mathrm{M}+\mathrm{H}]^{+} ; \text {Mesia et al, 2010 isomer } 1}\end{array}$ \\
\hline 12 & 3.15 & 0,79 & 185.1 & 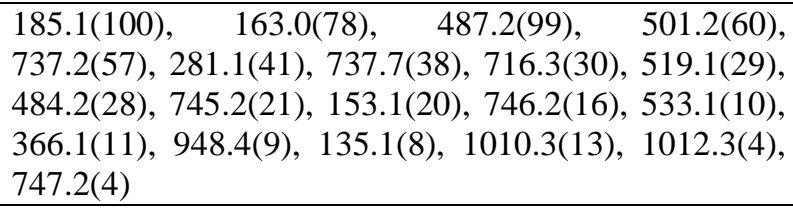 \\
\hline 13 & 3.30 & 1,47 & 533.2 & $\begin{array}{llr}533.2(100), & 182.1(55), \quad 534.2(29), & 179.1(20), \\
531.2(19), 211.1(17), 803.3(14), 542.2(11), & 545.2(5), \\
811.3(5), 281.1(4), 439.1(3), & 705.3(2), & 1064.4(3), \\
1100.3(3), 1326.0(1) & & \end{array}$ \\
\hline 14 & 3.35 & 1 & 533.2 & $\begin{array}{lccc}533.2(100), & 534.2(27), & 182.1(17), & 211.1(11.5), \\
542.2(8), 803.3(7), 281.1(4), & 1064.4(2) & \end{array}$ \\
\hline 15 & 3.54 & 1,24 & 543.2 & $\begin{array}{l}\text { 543.2(100), 559.2(27), 325.1(11), 181.1(9), 560.2(6), } \\
219.1(5), 327.2(2), 681.3(1)\end{array}$ \\
\hline 16 & 3.62 & 0,66 & 533.2 & $\begin{array}{l}533.2(100), \quad 227.1(67), \quad 501.2(26), \quad 209.1(24), \\
534.2(24), 199.1(17), 275.1(10), 181.1(7), 578.2(6), \\
375.1(5), 111.1(3), 663.2(2), 798.8(2), 1040.9(1)\end{array}$ \\
\hline 17 & 3.71 & 4 & 561.2 & $\begin{array}{lccc}561.2(100), & 577.2(91), & 578.2(24), & 533.2(21), \\
327.2(10), & 373.2(11), 227.2(9), & 501.2(4), & 579.2(4),\end{array}$ \\
\hline
\end{tabular}


Identification of New Molecules in the Trunk Bark of Sarcocephalus Pobeguini.

\begin{tabular}{|c|c|c|c|c|}
\hline & & & & 209.1(3), 275.1(2), 751.3(2) \\
\hline 18 & 3.87 & 0,35 & 517.2 & 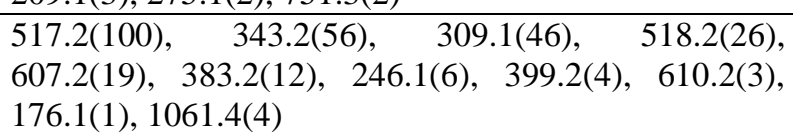 \\
\hline 19 & 4.05 & 1,73 & 589.2 & $\begin{array}{lccc}589.2(100), & 527.2(69), & 401.2(55), & 737.3(36), \\
590.2(31), 309.1(15), 402.2(13), 738.3(12), & 415.2(3), \\
739.3(3), 199.1(1), 591.2(1), 899.3(1) & \\
\end{array}$ \\
\hline 20 & 4.27 & 15 & 575.2 & $\begin{array}{l}\text { 575.2(100), 576.2(55), 577.2(22), 413.2(7), 558.2(6), } \\
\text { 323.1(4), 578.2(3), 168.1(1), 1149.4(1) } \\
\text { Pic 20 = 5-carboxystrictosidine ; } \mathrm{C}_{28} \mathrm{H}_{35} \mathrm{O}_{11} \quad \mathrm{~N}_{2} ; \\
{[\mathrm{M}+\mathrm{H}]^{+}=575.2 ; \text { Mesia et al, 2010; isomer } 2}\end{array}$ \\
\hline 21 & 4.37 & 9 & 513.2 & 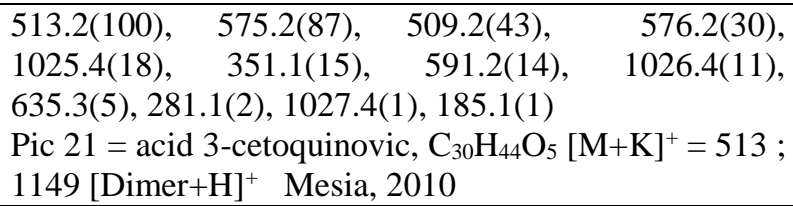 \\
\hline 22 & 4.52 & 7 & 555.2 & $\begin{array}{l}\text { 555.2(100), 556.2(43), 571.2(27), 576.2(30), }), \\
\text { 367.1(2), 293.1(1) } \\
\text { Pic 22 = strictosidine, } \mathrm{C}_{27} \mathrm{H}_{34} \mathrm{O}_{9} \mathrm{~N}_{2} ; \\
{[\mathrm{M}+\mathrm{Na}]^{+}=555 \mathrm{Xu} \text { et } \mathrm{al}, 2012 ;}\end{array}$ \\
\hline 23 & 4.63 & 3,21 & 494.2 & $\begin{array}{l}494.2(100), 513.2(84), 464.2(22), 555.2(21), 575.2(9), \\
589.3(3), 191.1(1), 293.1(2) 322.1(1), 1007.3(1)\end{array}$ \\
\hline 24 & 4.87 & 0,83 & 429.2 & 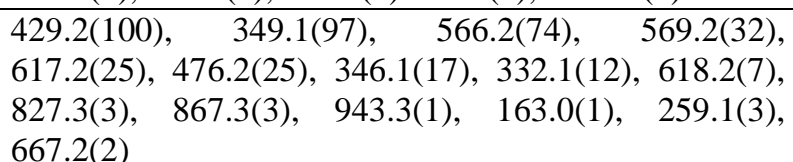 \\
\hline 25 & 4.98 & 0,28 & 349.1 & $\begin{array}{lccc}349.1(100), & 508.2(59), & 513.2(51), & 407.2(18), \\
566.2(16), \quad 346.2(11), & 573.2(5), & 665.2(4), & 163.0(2), \\
315.1(2), 797.4(1) & & & \end{array}$ \\
\hline 26 & 5.19 & 2,64 & 522.2 & $\begin{array}{lccc}522.2(100), \quad 523.2(30), \quad 411.2((24), & 418.2(14), \\
397.2(11), \quad 419.2(4), \quad 603.3(4), & 524.2(4), & 419.2(4), \\
264.1(1) 351.1(1), 725.3(1) & & \end{array}$ \\
\hline 27 & 5.27 & 4,01 & 543.2 & $\begin{array}{lccc}543.2(100), & 544.2(34), \quad 381.1(27), & 522.2(22), \\
411.2(21), & 661.3(7), 1085.4(3), & 662.3(3), & 379.1(2), \\
169.1(1), 291.1(1), 833.8(1) & \end{array}$ \\
\hline 28 & 5.41 & 1,23 & 411.2 & 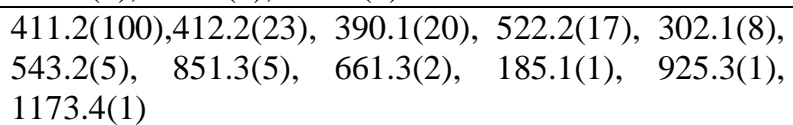 \\
\hline 29 & 5.48 & 0,88 & 332.1 & $\begin{array}{lccc}332.1(100), & 302.1(74), & 438.1(36), & 375.1(16), \\
288.1(11), 462.2(11), 522.2(5), & 851.3(5), & 210.1(1), \\
631.3(1), 947.3(1) & 499.2(100), 997.4(93), & 998.4(65), \\
337.2(56), 500.2 \quad(46), \quad 999.4(21), & 338.2(18), \\
267.1(10), 171.1(9), \quad 501.2(9), & 1000.4(4), & 521.2(2), \\
1266.0(2), 172.1(1), 767.8(1) & & \\
\text { Pic 30 }=\text { Strictosamide } ; \mathrm{C}_{26} \mathrm{H}_{30} \mathrm{O}_{8} \mathrm{~N}_{2} & \end{array}$ \\
\hline 30 & 5.78 & 12 & 499.2 & $\begin{array}{lll}499.2(100), 997.4(93), 998.4(65), & \\
337.2(56), 500.2 \quad(46), \quad 999.4(21), & 338.2(18), \\
267.1(10), 171.1(9), \quad 501.2(9), & 1000.4(4), & 521.2(2), \\
1266.0(2), 172.1(1), 767.8(1) & & \end{array}$ \\
\hline 31 & 5.98 & 1,26 & 469.3 & $\begin{array}{l}469.3(100), 499.2(59), 500.2(15), 337.2(12), 307.7(8), \\
817.4(5), 583.3(3), 679.3(2), 289.1(1), 1108.6(1)\end{array}$ \\
\hline 32 & 6.15 & 4 & 469.3 & $\begin{array}{l}469.3(100), \quad 470.3(33), \quad 423.3(26), \quad 487.3(14), \\
341.2(14), \quad 817.4(5), \quad 316.1(5), \quad 488.4(5), 191.2(1), \\
671.4(1), 769.4(1), 958.5(1), 1212.2(1)\end{array}$ \\
\hline 33 & 6.29 & 5,04 & 499.2 & $\begin{array}{l}\text { 499.2(100),543.2(93), 381.1(30), 544.2(25), 496.2(11), } \\
334.1(10), \quad 382.1(6), 545.2(4), \quad 1085.4(4), 307.1(2), \\
907.3(2), 171.1(1) 817.3(1)\end{array}$ \\
\hline 34 & 6.49 & 0,1 & 379.2 & $\begin{array}{lccc}379.2(100), & 599.2(60), & 339.2(48), & 391.1(23), \\
600.2(18), 480.2(17), & 305.1(13), 290.1(5), & 571.8(3),\end{array}$ \\
\hline
\end{tabular}


Identification of New Molecules in the Trunk Bark of Sarcocephalus Pobeguinii.

\begin{tabular}{|c|c|c|c|c|}
\hline & & & & 899.3(3), 601.2(2), 1120.6(2), 221.1(1), 776.3(1) \\
\hline 35 & 6.58 & 0,20 & 599.2 & $\begin{array}{l}599.2(100), \quad 541.2(73), \quad 321.2(68), \quad 413.2(60), \\
265.1(53), 859.3(22), 223.1(9), 524.2(13), 906.4(6), \\
1186.6(5), 1023.5(3), 152.0(2), 830.9(1), 1469.3(1) \\
\text { Peak 35 = tetrahydrodéoxycordifoline ; }[\mathrm{M}+\mathrm{Na}]^{+}= \\
599 ; \mathrm{C}_{28} \mathrm{H}_{36} \mathrm{O}_{11} \mathrm{~N}_{2}\end{array}$ \\
\hline 36 & 6.68 & 0,17 & 321.1 & 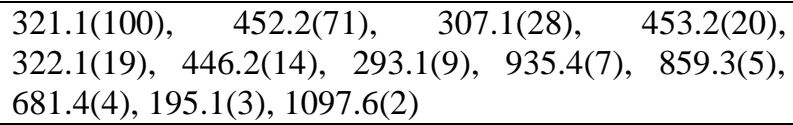 \\
\hline 37 & 6.87 & 0,62 & 303.1 & $\begin{array}{l}\text { 303.1(100), 331.1(42), 335.1(23), 787.3(8), 336.1(4), } \\
\text { 788.3(4), 519.2(4) } \\
\text { Peak 37 = Quercetin ; }[\mathrm{M}+\mathrm{H}]^{+}=303 \\
\mathrm{C}_{15} \mathrm{H}_{10} \mathrm{O}_{7}\end{array}$ \\
\hline 38 & 6.92 & 0,48 & 321.2 & 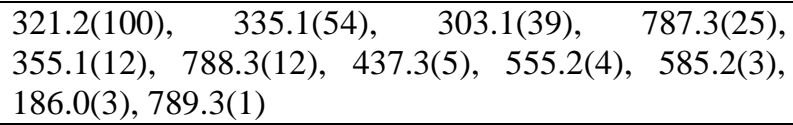 \\
\hline 39 & 7.13 & 2 & 360.2 & $\begin{array}{l}360.2(100), 425.4(15), 337.2(13), 930.5(14), 476.2(6), \\
856.3(4), 936.5(6), \quad 817.4(2), \quad 288.3(1), \\
617.3(1), 953.5(1)\end{array}$ \\
\hline 40 & 7.36 & 0,54 & 337.2 & $\begin{array}{l}\text { 337.2(100), 361.2(38), 362.2(4), 468.1(3),606.3(2), } \\
\text { 208.6(1), 288.3(1), 861.3(1) } \\
\text { Peak 40 = magniflorine ; }[\mathrm{M}+\mathrm{H}]^{+}=377 ; \mathrm{C}_{20} \mathrm{H}_{20} \mathrm{O}_{3} \mathrm{~N}_{2}\end{array}$ \\
\hline 41 & 7.47 & 0,27 & 383.2 & 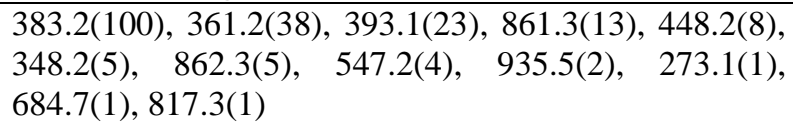 \\
\hline 42 & 7.60 & 1,5 & 831.3 & $\begin{array}{lccc}831.3(100), & 832.3(48), \quad 330.1(40), & 315.1(25), \\
359.1(11), \quad 451.3(9), \quad 313.1(3), & 833,3(7), & 497.3(2), \\
728.3(2), 834.3(1) & & & \end{array}$ \\
\hline 43 & 7.70 & 2 & 361.2 & $\begin{array}{l}\text { 361.2(100), 362.1(22), 316.1(6), 363.2(2), 508.2(1), } \\
575.2(1), 831.3(1), 859.3(1)\end{array}$ \\
\hline 44 & 7.77 & 0,41 & 361.2 & 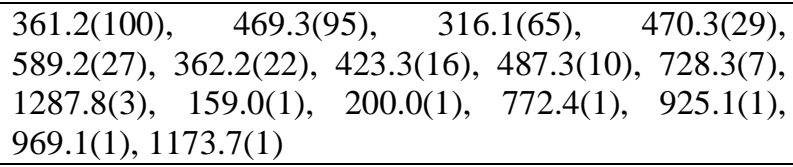 \\
\hline 45 & 7.92 & 0,37 & 362.2 & $\begin{array}{l}\text { 362.2(100), 383.1(20), 363.2(18), 1108.6(3), 364.2(2), } \\
291.1(1), 439.1(1), 588.2(1)\end{array}$ \\
\hline 46 & 8.04 & 0,21 & 425.4 & $\begin{array}{l}\begin{array}{l}425.4(100), \\
773.4(26), 485.1(23), 335.1(18), 636.2(14),\end{array} \text { 774.5(10), } \\
486.1(6), 790.9(3), 273.2(3), 191.2(2), 1362.8(1)\end{array}$ \\
\hline 47 & 8.19 & 0,83 & 331.1 & $\begin{array}{l}\text { 331.1(100), 332.1(20), 333.1(13), 335.1(8), 353.1(3), } \\
\text { 307.1(2) }\end{array}$ \\
\hline 48 & 8.31 & 0,55 & 331.1 & $\begin{array}{l}\text { 331.1(100), 332.1(21), 305.1(5), 619.2(2), 347.1(1), } \\
\text { 551.1(1) }\end{array}$ \\
\hline 49 & 8.73 & 0,43 & 315.1 & $\begin{array}{l}\text { 315.1(100), 314.1(30), 316.1(19), 319.1(5), 177.0(1), } \\
\text { 454.2(1),625.3(1) }\end{array}$ \\
\hline 50 & 8.87 & 2 & 319.1 & $\begin{array}{l}319.1(100), 361.2(23), 362.2(6), 303.1(4), 485.3(2), \\
617.2(2)\end{array}$ \\
\hline 51 & 9.04 & 0,18 & 361.2 & $\begin{array}{l}\text { 361.2(100), 362.2(22), 425.4(10), 773.5(4), 426.4(3), } \\
287.2(1), 633.2(1)\end{array}$ \\
\hline
\end{tabular}

The coupling of UPLC to ionization mode mass spectrometry allowed the identification of quercetin (peak no. 30 with $[\mathrm{M}+\mathrm{H}]+=303$; C15H10 O7), magniflorine (peak no. $40=$ with $[\mathrm{M}+\mathrm{H}]+=377$; $\mathrm{C} 20 \mathrm{H} 20 \mathrm{O} 3 \mathrm{~N} 2$ ) and tetrahydrodeoxycordifoline (Peak no. $35=;[\mathrm{M}+\mathrm{Na}]+=599 ; \mathrm{C} 28 \mathrm{H} 36 \mathrm{O} 11 \mathrm{~N} 2)$ in the trunk bark of Sarcocephalus pobeguinii.

Analysis of trunk bark of Sarcocephalus pobeguinii by HPLC coupled with mass spectroscopy allowed the identification and isolation of quercetin, magniflorin and tetrahydrodeoxycordifoline. Magnlorin and tetrahydrodeoxycordifoline are alkaloid compounds and quercetin are a tannin. These results corroborate those of other researchers who had identified in trunk bark in addition to (5S)-5carboxystrictosidine, 19-O-methylangustoline and strictosamide, 3-O- $\beta$-fucosylquinovic acid and 3- 
ketoquinovic acid [20]. Further work has shown that some alkaloids and other compounds have been found in other plant organs. Thus, other alkaloids such as angustine, naufoline, angustoline, nauclefine, O-acetyl-angustoline, 3,14-dihydroangustine as well as two quinovic acid glycosides have been identified in the roots by authors $[16,17,18,21,22]$. Similarly, naucleamides A, B, C, D, E and F, nauclefolinin, angustin, angustifolin, nauclefin and naucletin, naulafin, 3--dihydrocadambin, cadambin and naufolin, naucleidinal and epinauclédinal, isovincoside lactam (strictosidine lactam or strictosamide), 10-hydroxystrictosamide, nauclefoline and nauclechine have also been isolated from stem bark and various other organs [7,12,19,20,23].

\section{CONClusion}

Quercetin, magniflorin and tetrahydrodeoxycordifoline were detected in the trunk bark of Sarcocephalus pobeguinii by HPLC coupled with mass spectroscopy. These results require further fractionation in order to isolate the active ingredients.

\section{ACKNOWLEDGEMENT}

We thank the University of Sciences and Techniques of Masuku of Gabon and the University of Bamako of Mali for their funding and Issembé Yves and Niangadouma for their assistance in plant collection and identification.

\section{REFERENCES}

[1] Asase A. ; Oppong M.G. Remèdes traditionnels de phytothérapie antipaludique dans les marchés de plantes médicinales du sud du Ghana. Journal of Ethnopharmacology, 2009, 126, 492-499.

[2] Mesia G.K. ; Cimanga R.K. ; Dhooghe L. ; Cos P. ; Apers S. ; Totte J. ; Tona G.L. ; Pieters L. ; Vlietinck A.J. ; Maes L. Antimalarial activity and toxicity evaluation of a quantified Nauclea pobeguinii extract. Journal of Ethnopharmacology, 2010 13, 10-16.

[3] Mesia K. ; Tona L., Mampunza M.M. ; Ntamabyaliro N. ; Muanda T. ; Muyembe T. ; Cimanga K. ; Totte J. ; Mets T. ; Pieters L. Antimalarial effectiveness of a quantified extract of Nauclea pobeguinii stem bark chez des volontaires adultes humains chez qui on a diagnostiqué un paludisme à falciparum non compliqué. Partie 1 : un essai clinique de phase IIA Planta medica, 2012a , 78(3), 211-218.

[4] Mesia K. ; Tona L., Mampunza M.M. ; Ntamabyaliro N. ; Muanda T. ; Muyembe T. ; Cimanga K. ; Totte J. ; Mets T. ; Pieters L. Efficacité antipaludique d'un extrait quantifié d'écorce de Nauclea pobeguinii chez des volontaires adultes humains atteints de paludisme à falciparum non compliqué. Partie 2 : un essai clinique de phase IIB Planta medica, 2012b, 78(9), 853-860.

[5] Seukep A.J. ; Louis P.S. ; Bonaventure T.N. ; Victor K. Antibacterial and antibiotic-resistance modifying activity of the extracts and compounds from Nauclea pobeguinii against Gram-ngative multi-drug resistant phenotypes, BMC , 2016, 16:193.

[6] Kadari H. ; Adegor E. ; Asgba S.O. Effet d'un extrait aqueux de feuilles de Nauclea pobeguinii sur des rats ayant subi des lésions hépatiques. Res J Med Plant. 2007, 1 : 139-143.

[7] Shigemori H. ; Kagata T. ; Ishiyama H. ; Morah F. ; Ohsaki A. ; Kobayashi J. Naucleamides A-E, nouveaux alcaloïdes indole monoterpènes de Nauclea pobeguinii. Chimie. Pharm. Bull, 2003, 51 : 58-61.

[8] Traore K. F. ; Gasquet M. ; Di-giorgio C. ; Ollivier E., Delmas F. Activité antipaludique de quatre plantes utilisées dans la médecine traditionnelle au Mali. Phytothérapeute. Res., 2000, 14 : 45-47.

[9] Singla A.K ; Pathak K. Phytochimie des espèces d'euphorbes, Fitoterapia, LXI (6), 1990, 483- 516,

[10] Lamidi M. ; Ollivier E. ; Faure R. ; Debrauwer L. ; Nze E.L. ; Balansard G. Trois saponines ont été isolées des tiges de Nauclea diderrichii et leurs structures établies. Ces saponines sont décrites pour la première fois dans cette plante. L'acide quinovique 3-O--L-rhamnosyl (28-5)-Tétrahydrodesoxycordifoline de Nauclea diderrichii (de Wild) Merr. Bark. Pharm Pharmacol. Lettres. 1995, 5(1), 8-9.

[11] Lamidi M. ; Olllivier E. ; Mahiou V. ; Faure R. ; Debrauwer L. ; Nze E.L. ; Balansard G. Alcaloïdes de gluco-indole provenant de l'écorce de Nauclea diderrichii. Affectations RMN $1 \mathrm{H}$ et $13 \mathrm{C}$ du $3 \mathrm{a}-5 \mathrm{a}-$ tétrahydrodésoxycordifoline lactame et de l'acide cadambine. Magn. Réson. Chim. 2005, 43, 427-429

[12] Kakuguchi Y. ; Ishiyama H. ; Kubota T. ; Kobayashi J. Naucleamide F, un nouvel alcaloïde indole monoterpène de Nauclea latifolia. Heterocycles, 2009, 79, 765-771.

[13] Ngnokam D. ; Ayafor J.F. ; Connolly J.D. ; Nuzillard J.M. Nauclefolinine : un nouvel alcaloïde provenant des racines de Nauclea latifolia. Bulletin de la Société chimique d'Éthiopie, 2003, 17(2), 173-176.

[14] Sook Y.L. ; Mat R.M. ; Hamid A.A.H. ; Khalijah A. ; Mohd R.M. ; Kazumasa Z., Hiroshi M. ; Marc L. Naucline, un nouvel alcaloïde indole issu de l'écorce de Nauclea officinalis. Molecules, 2012, 17, 40284036. 
[15] Zeches M. ; Richard B. ; Gueye M.L. ; Le M.O.L. ; Delaude C. Neuf constituants ont été isolés à partir de l'écorce de la racine de Nauclea pobeguinii. Il s'agit du strictosamide connu comme constituant principal ; de 2 glycosides de l'acide quinovique et de 4 alcaloïdes connus, l'angustine, la naufoline, l'angustoline, la nauclefine ; et de 2 nouveaux alcaloïdes, la O-acétylangusatoline et la 3,14-dihydroangustine. J Natur Prod. 1985, 48(1), 42-46.

[16] Anam E.M. Nouvelle nauclequiniine provenant de l'extrait de racine de Nauclea pobeguinii (Pob. \& Pellegr.) Petit (Rubiaceae). Ind. J. Chem, Section B : Chimie organique, y compris la chimie médicinale, 36B (1), 1997, 54-56.

[17] Brown R.T. ; Chapple C.L. ; Lashford A.G. Isolation of strictosidine (isovincoside) lactam from Nauclea latifolia. Phytochimie (Elsevier), 1977, 16(10), 1619-1620.

[18] Xu Y.J. ; Foubert K. ; Dhooghe L. ; Lemiere F. ; Cimanga K. ; Mesia K. ; Apers S., Pieters L. Profilage chromatographique et identification de deux nouveaux alcaloïdes de l'indole iridoide par UPLC-MS et HPLC-SPE-NMR d'un extrait antipaludique de Nauclea pobeguinii. Phytochem. Lett. 2012, 5(2), 316-319.

[19] Agomuoh A.A., Ata A., Udenigwe C.C., Aluko R.E., Irenus I. Nouveaux alcaloïdes indole de Nauclea latifolia et leurs activités inhibitrices de la rénine Chimie et biodiversité, 2013, 10(3), 401-10.

[20] Kahumu M. ; Richard K. C. ; Liene D. ; Paul C. ; Sandra A. ; Jozef T. ; Gaston L.T. ; Luc P., Arnold J.V., Louis M. Antimalarial activity and toxicity evaluation of a quantified Nauclea pobeguinii extract. J Ethnopharmacol. 2010, vol. 131, no1, p. 10-16.

[21] Dimitrienko G.I., Szakolcai A., Lean S. Constituants de Nauclea diderrichii. VI. Naucleonine et naucleonidine Tétraèdre Lettres, 1974 , (30), 2599-2602.

[22] Adeoye A.O., Waigh R.D. Secoiridoid and triterpenic acids from the stems of Nauclea diderrichii. Phytochemistry (Elsevier), 1983, 22(4), 975-978.

[23] Sun J., Lou H., Dai S., Xu H., Zhao F., Lui K. Indole alkaloids from Nauclea officinalis with weak antimalarial activity. Phytochemistry (Elsevier), 2008, 69(6), 1405-1410.

Citation: Ousmane Kéita, et.al., (2020). Identification of New Molecules in the Trunk Bark of Sarcocephalus Pobeguinii. International Journal of Medicinal Plants and Natural Products (IJMPNP), 6(1), pp.15-21. http://dx.doi.org/ 10.20431/2454-7999.0601004

Copyright: () 2020 Authors, this is an open-access article distributed under the terms of the Creative Commons Attribution License, which permits unrestricted use, distribution, and reproduction in any medium, provided the original author and source are credited. 